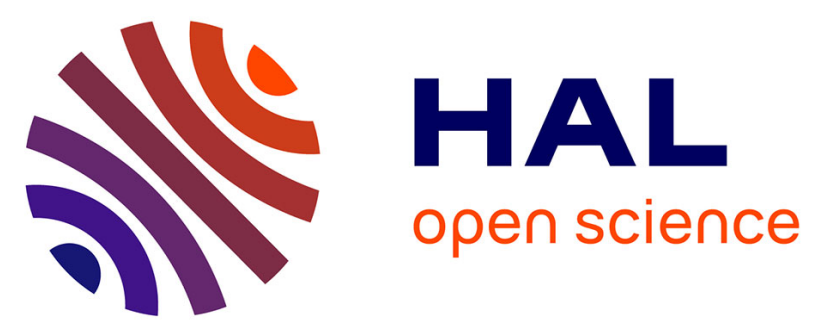

\title{
Dual Polarized Versus Single Polarized MIMO: A Study Over NLOS Propagation with Polarization Discrimination and Spatial Correlation Effects
}

\author{
Maha Ben Zid, Kosai Raoof, Ammar Bouallegue
}

\section{- To cite this version:}

Maha Ben Zid, Kosai Raoof, Ammar Bouallegue. Dual Polarized Versus Single Polarized MIMO: A Study Over NLOS Propagation with Polarization Discrimination and Spatial Correlation Effects. EuCAP 2012 - 6th European Conference on Antennas and Propagation, Mar 2012, Prague, Czech Republic. pp.n/a. hal-00690605

\section{HAL Id: hal-00690605 https://hal.science/hal-00690605}

Submitted on 24 Apr 2012

HAL is a multi-disciplinary open access archive for the deposit and dissemination of scientific research documents, whether they are published or not. The documents may come from teaching and research institutions in France or abroad, or from public or private research centers.
L'archive ouverte pluridisciplinaire HAL, est destinée au dépôt et à la diffusion de documents scientifiques de niveau recherche, publiés ou non, émanant des établissements d'enseignement et de recherche français ou étrangers, des laboratoires publics ou privés. 


\title{
Dual Polarized Versus Single Polarized MIMO: A Study Over NLOS Propagation with Polarization Discrimination and Spatial Correlation Effects
}

\author{
Maha Ben Zid*,‡, Kosai Raoof ${ }^{\dagger}$, and Ammar Bouallegue $\ddagger$ \\ * University of Grenoble \\ Grenoble Images Parole Signal Automatique (GIPSA-Lab), UMR-CNRS 5216 \\ 11 rue des Mathématiques Grenoble Campus, BP 46, 38402 Saint Martin d'Hères Cedex \\ Grenoble, France \\ Email: maha.ben-zid@gipsa-lab.grenoble-inp.fr \\ $\dagger$ University of Maine \\ National School of Engineering of Le Mans (ENSIM), LAUM, UMR-CNRS 6613 \\ rue Aristote 72085 \\ Le Mans Cedex 09, France \\ Email: kosai.raoof@univ-lemans.fr \\ $\ddagger$ National Engineering School of Tunis (ENIT) \\ 6'COM-Lab \\ Le Belvédère. BP 37, 1002 \\ Tunis, Tunisia \\ Email: ammar.bouallegue@enit.rnu.tn
}

\begin{abstract}
We investigate in this paper the use of dual polarized antennas in Multiple Input Multiple Output (MIMO) systems[1][2]. Among the existing channel models for MIMO systems, we perform the geometric NLOS (Non Line of Sight) propagation channel model. Our analysis evaluates the gain in capacity of a MIMO system with two transmit antennas and two receive antennas when comparing the capacity of the dual polarized MIMO system to the capacity of MIMO system with single polarized antennas. The effect of the antenna depolarization as well as the spatial correlation of antennas are considered when evaluating the capacity gain.
\end{abstract}

\section{INTRODUCTION}

Antenna polarization has been proposed as an attractive strategy for MIMO systems. Advanced polarization techniques such as dual antennas polarization have been shown to improve the channel spectral efficiency of multiple antennas systems when comparing to the classical MIMO systems. Rigorous channel modeling for MIMO systems should be considered for propagation channel with rich scatterers where mismatch in antenna polarization could occur. The presence of scatterers results in the angular spreading phenomena which impacts the spatial correlation of antennas. In this paper, we investigate the use of dual polarized MIMO systems over NLOS propagation with polarization discrimination and spatial correlation effects. The remainder of this paper proceeds as follows. Section II presents the geometric channel model for the performed
MIMO system with polarized antennas. Section III is attempted to address the problem of antenna depolarization in rich scattering environment. Channel modeling involving the depolarization effect is then introduced. Section IV is devoted to evaluate the spatial correlation between antennas over the wide band channel propagation. Besides spatial correlation is derived, section $\mathrm{V}$ provides the simulation results of the spatial correlation for both the single and the dual polarized MIMO systems. The gain in capacity that could be achieved via dual polarization strategy is evaluated as a function of the spatial correlation and the cross polarization discrimination. Finally, we present our concluding remarks in section VI

\section{COMMUNiCATION SYSTEM MODEL}

The communication system model is presented in Fig.1. The performed model falls into the class of the GeometricBased Stochastic Channel Models (GSCMs). Two antennas are implemented at both the transmit side and the receive side of the communication link. Transmit antennas are denoted by $A_{\mathrm{Tx}}^{(1)}$ and $A_{\mathrm{Tx}}^{(2)}$. The receive antennas are denoted by $A_{\mathrm{Rx}}^{(1)}$ and $A_{\mathrm{Rx}}^{(2)}$. We assume that scatterers are distributed in the propagation environment. Clusters of scatterers are considered around both the transmitter and the receiver. Clusters of index $\ell ; \quad \ell=1, \ldots, L$ around the transmitter and the receiver are respectively denoted by $C_{\mathrm{Tx}}^{(\ell)}$ and $C_{\mathrm{Rx}}^{(\ell)}$ where $L$ denotes the number of clusters. 
$C_{\mathrm{Tx}}^{(\ell)}$ contains $M$ scatterers $S_{\mathrm{Tx}}^{(\ell, m)} ; \quad m=1, \ldots, M$ and $N$ scatterers $S_{\mathrm{Rx}}^{(\ell, n)} ; \quad n=1, \ldots, N$ are assumed to be within the cluster $C_{\mathrm{Rx}}^{(\ell)}$. In the following, we only consider one cluster of scatterers at each end of the communication link. The index $\ell$ will be omitted.

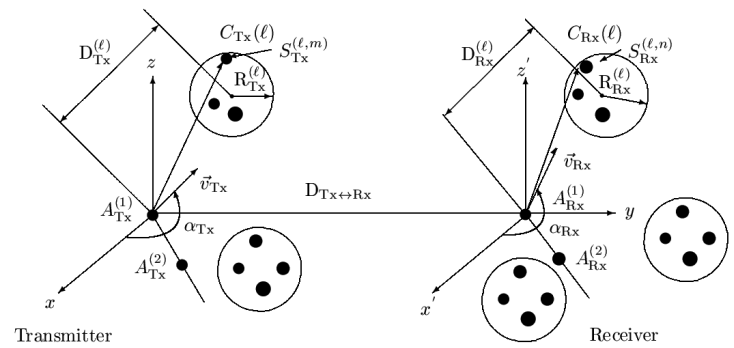

Fig. 1. MIMO channel model

We take for notations:

- $\mathrm{R}_{\mathrm{Tx}}$ : Transmit cluster radius

- $\mathrm{D}_{\mathrm{Tx}}$ : Distance between the reference transmit antenna and the transmit cluster center

- $d_{\mathrm{Tx}}$ : Transmit antennas spacing

- $d_{\mathrm{Rx}}$ : Receive antennas spacing

- $\mathrm{D}_{\mathrm{Tx} \leftrightarrow \mathrm{Rx}}$ : Distance between the transmitter and the receiver

- $\mathrm{R}_{\mathrm{Rx}}$ : Cluster radius at the receiver

- $\mathrm{D}_{\mathrm{Rx}}$ : Distance between the reference antenna $A_{\mathrm{Rx}}^{(1)}$ and the center of the cluster at the receiver.

- $\vec{v}_{\mathrm{Tx}}$ : Velocity of the transmitter.

- $\vec{v}_{\mathrm{Rx}}$ : Velocity of the receiver.

- The transmitter and the receiver have motions above the plan $(x, y)$ with relative directions $\alpha_{\mathrm{Tx}}$ and $\alpha_{\mathrm{Rx}}$.

\section{ANTENNA DEPOLARIZATION}

The polarization of the electric field is defined by its direction and its magnitude. In rich scattering environment, the electric field components are reflected. In matrix notations, the reflected electric field represented by $E_{r}$ is expressed as the product of the scattering matrix $S$ and the radiation pattern $E_{i}$ as given by equation (1).

$$
E_{r}=\mathrm{S} \times \mathrm{E}_{i}
$$

where:

$$
E_{i}=\left[\begin{array}{c}
E_{i, \theta} \\
E_{i, \phi}
\end{array}\right]
$$

$E_{i}$ is expressed as a function of the azimuth and the elevation amplitudes of polarization vectors in both the elevation and the azimuth directions $\hat{\theta}$ and $\hat{\phi}$. The azimuth and the elevation amplitudes are respectively denoted by $E_{i, \theta}$ and $E_{i, \phi}$.

- The matrix notation for the reflected electric field is expressed as:

$$
E_{r}=\left[\begin{array}{c}
E_{r, \theta} \\
E_{r, \phi}
\end{array}\right]
$$

$E_{r, \theta}$ and $E_{r, \phi}$ are respectively the reflected elevation and azimuth components of the incident electric field $E_{i}$.

- $\mathrm{S}$ is the scattering matrix given by:

$$
\mathrm{S}=\left[\begin{array}{ll}
v_{\theta \theta} & v_{\theta \phi} \\
v_{\phi \theta} & v_{\phi \phi}
\end{array}\right]
$$

where:

- $v_{\theta \theta}$ is the co-polarization gain of the elevation transmission.

- $v_{\phi \phi}$ is the co-polarization gain of the azimuth transmission.

- $v_{\theta \phi}$ is the depolarization gain of the elevation polarization relative to the azimuth polarization.

- $v_{\phi \theta}$ is the depolarization gain of the azimuth polarization relative to the elevation polarization.

Scattering phenomena results in the depolarization effect which could be evaluated by the cross polarization discrimination (XPD)[3][4][5].

We distinguish the cross polarization discrimination for the elevation transmission and cross polarization discrimination for the azimuth transmission which are respectively given by equation (5) and equation (6).

$$
\begin{aligned}
X P D_{\theta} & =\frac{\mathrm{E}\left|v_{\theta \theta}\right|^{2}}{\mathrm{E}\left|v_{\phi \theta}\right|^{2}} \\
X P D_{\phi} & =\frac{\mathrm{E}\left|v_{\phi \phi}\right|^{2}}{\mathrm{E}\left|v_{\theta \phi}\right|^{2}}
\end{aligned}
$$

In the following we denote:

$$
\chi_{\theta}=\frac{1}{X P D_{\theta}}
$$

and

$$
\chi_{\phi}=\frac{1}{X P D_{\phi}}
$$

The scattering matrix relative to the $m t h$ scatterer at the the transmit side and the $n t h$ scatterer at the receive side when considering the depolarization effect is denoted by $\mathrm{S}_{\mathrm{Tx}, \mathrm{Rx}}^{(m, n)}$ and is expressed as:

$\mathrm{S}_{\mathrm{Tx}, \mathrm{Rx}}^{(m, n)}=\left[\begin{array}{cc}\sqrt{\frac{1}{1+\chi_{\theta}^{(m, n)}} e^{j \varepsilon_{\theta \theta}^{(m, n)}}} & \sqrt{\frac{\chi_{\phi}^{(m, n)}}{1+\chi_{\phi}^{(m, n)}}} e^{j \varepsilon_{\theta \phi}^{(m, n)}} \\ \sqrt{\frac{\chi_{\theta}^{(m, n)}}{1+\chi_{\theta}^{(m, n)}}} e^{j \varepsilon_{\phi \theta}^{(m, n)}} & \sqrt{\frac{1}{1+\chi_{\phi}^{(m, n)}}} e^{j \varepsilon_{\phi \phi}^{(m, n)}}\end{array}\right]$

where:

$\varepsilon_{\theta \theta}^{(m, n)}, \varepsilon_{\theta \phi}^{(m, n)}, \varepsilon_{\phi \theta}^{(m, n)}$ and $\varepsilon_{\phi \phi}^{(m, n)}$ are the phase offsets.

We finally express the wide band GSCM channel coefficients $h_{q p}^{N L O S} ; \quad q \in\left\{1, \ldots, N_{R}\right\}, p \in\left\{1, \ldots, N_{T}\right\}$ in terms of time and frequency [7] by equation (7).

The number of receive antennas and the number of transmit antennas are respectively denoted by $N_{R}$ and $N_{T}$. 
In this paper, the number of antennas at both the ends of the communication link are set to 2 .

$$
\begin{aligned}
& h_{q p}^{N L O S}(t, f)=\lim _{M, N \rightarrow \infty} \sqrt{\frac{P D P}{M N}} \sum_{m=1}^{M} \sum_{n=1}^{N} a_{m}^{(p)} b_{n}^{(q)} \\
& \times \exp \left\{j\left(2 \pi\left(f_{\mathrm{Tx}}^{(m)}+f_{\mathrm{Rx}}^{(n)}\right) t+\varphi_{m, n}+\varphi_{0}\right)\right\} \\
& \times\left[\begin{array}{c}
G_{q, \theta} \\
G_{q, \phi}
\end{array}\right]^{T} \mathrm{~S}_{\mathrm{Tx}, \mathrm{Rx}}^{(m, n)}\left[\begin{array}{c}
G_{p, \theta} \\
G_{p, \phi}
\end{array}\right](7)
\end{aligned}
$$

- PDP denotes the power delay profile.

- $a_{m}^{(p)}$ is the transmitting steering vector expressed as:

$$
\begin{aligned}
a_{m}^{(p)} & =\exp \left\{j 2 \pi ( p - 1 ) ( d _ { \mathrm { Tx } } / \lambda ) \left[\cos \left(\theta_{\mathrm{Tx}}^{(m)}\right) \cos \left(\beta_{\mathrm{Tx}}\right)\right.\right. \\
& \left.\left.+\sin \left(\theta_{\mathrm{Tx}}^{(m)}\right) \sin \left(\beta_{\mathrm{Tx}}\right) \cos \left(\phi_{\mathrm{Tx}}^{(m)}-\gamma_{\mathrm{Tx}}\right)\right]\right\}
\end{aligned}
$$

- $b_{n}^{(q)}$ is the receiving steering vector expressed as:

$$
\begin{aligned}
b_{n}^{(q)} & =\exp \left\{j 2 \pi ( q - 1 ) ( d _ { \mathrm { Rx } } / \lambda ) \left[\cos \left(\theta_{\mathrm{Rx}}^{(n)}\right) \cos \left(\beta_{\mathrm{Rx}}\right)\right.\right. \\
& \left.\left.+\sin \left(\theta_{\mathrm{Rx}}^{(n)}\right) \sin \left(\beta_{\mathrm{Rx}}\right) \cos \left(\phi_{\mathrm{Rx}}^{(n)}-\gamma_{\mathrm{Rx}}\right)\right]\right\}
\end{aligned}
$$

- The frequency for the transmitter relative to scatterer of index $m$ is:

$$
f_{\mathrm{Tx}}^{(m)}=\left(\left|\vec{v}_{\mathrm{Tx}}\right| / \lambda\right) \sin \left(\theta_{\mathrm{Tx}}^{(m)}\right) \cos \left(\phi_{\mathrm{Tx}}^{(m)}-\alpha_{\mathrm{Tx}}\right)
$$

$\lambda$ denotes the wavelength.

- The frequency for the transmitter relative to scatterer of index $m$ is:

$$
f_{\mathrm{Rx}}^{(n)}=\left(\left|\vec{v}_{\mathrm{Rx}}\right| / \lambda\right) \sin \left(\theta_{\mathrm{Rx}}^{(n)}\right) \cos \left(\phi_{\mathrm{Rx}}^{(n)}-\alpha_{\mathrm{Rx}}\right)
$$

- The dephasing terms $\varphi_{0}$ and $\varphi_{m, n}$ are given by: $-\varphi_{0}=-2 \pi\left(\mathrm{D}_{\mathrm{Tx}}+\mathrm{D}_{\mathrm{Tx} \leftrightarrow \mathrm{Rx}}+\mathrm{D}_{\mathrm{Rx}}\right) / \lambda$ $-\varphi_{m, n} \sim U[-\pi, \pi]$

- $G_{q, \theta}\left(\theta_{\mathrm{Rx}}^{(n)}, \phi_{\mathrm{Rx}}^{(n)} ; \beta_{\mathrm{Rx}}, \gamma_{\mathrm{Rx}}\right)$ is the gain of the elevation component for antenna $q$ with associated oriented direction $\left(\beta_{\mathrm{Rx}}, \gamma_{\mathrm{Rx}}\right)$ and a wave propagation direction $\left(\theta_{\mathrm{Rx}}^{(n)}, \phi_{\mathrm{Rx}}^{(n)}\right)$

- $G_{q, \phi}\left(\theta_{\mathrm{Rx}}^{(n)}, \phi_{\mathrm{Rx}}^{(n)} ; \beta_{\mathrm{Rx}}, \gamma_{\mathrm{Rx}}\right)$ is the gain of the azimuth component for antenna $q$ with associated oriented direction $\left(\beta_{\mathrm{Rx}}, \gamma_{\mathrm{Rx}}\right)$ and a wave propagation direction $\left(\theta_{\mathrm{Rx}}^{(n)}, \phi_{\mathrm{Rx}}^{(n)}\right)$

- $G_{p, \theta}\left(\theta_{\mathrm{Tx}}^{(m)}, \phi_{\mathrm{Tx}}^{(m)} ; \beta_{\mathrm{Tx}}, \gamma_{\mathrm{Tx}}\right)$ is the gain of the elevation component for antenna $p$ with associated oriented direction $\left(\beta_{\mathrm{Tx}}, \gamma_{\mathrm{Tx}}\right)$ and a wave propagation direction $\left(\theta_{\mathrm{Tx}}^{(m)}, \phi_{\mathrm{Tx}}^{(m)}\right)$.

- $G_{p, \phi}\left(\theta_{\mathrm{Tx}}^{(m)}, \phi_{\mathrm{Tx}}^{(m)} ; \beta_{\mathrm{Tx}}, \gamma_{\mathrm{Tx}}\right)$ is the gain of the azimuth component for antenna $p$ with associated oriented direction $\left(\beta_{\mathrm{Tx}}, \gamma_{\mathrm{Tx}}\right)$ and a wave propagation direction $\left(\theta_{\mathrm{Tx}}^{(m)}, \phi_{\mathrm{Tx}}^{(m)}\right)$.

\section{ChanNEL CORRELATION}

We evaluate in this section the correlation coefficients when considering the channel coefficients $h_{q p}^{N L O S}(t, \tau)$ and $h_{\tilde{q} \tilde{p}}^{N L O S}(t, \tau)$. The correlation coefficient $R_{q p, \tilde{q} \tilde{p}}$ is given by:

$$
R_{q p, \tilde{q} \tilde{p}}\left(\xi, \tau-\tau^{\prime}\right)=\mathrm{E}\left[h_{q p}(t, \tau) h_{\tilde{q} \tilde{p}}^{*}\left(t-\xi, \tau^{\prime}\right)\right]
$$

where:

- $\mathrm{E}(\cdot)$ is the expectation operator.

- $(\cdot)^{*}$ is the conjugate operator.

We assume that:

$$
\chi_{\theta}=\chi_{\phi}=\chi
$$

The correlation coefficients $R_{q p, \tilde{q} \tilde{p}}(\xi)$ are obtained as a function of the product of three terms listed as follows:

1) Transmit spatial correlation: $R_{\cdot p, \cdot \tilde{p}}(\xi)$

$$
\begin{aligned}
& R_{\cdot p, \tilde{p}}(\xi)=\iint\left[\begin{array}{c}
G_{p, \theta} \cdot G_{\tilde{p}, \theta} \\
G_{p, \phi} \cdot G_{\tilde{p}, \phi}
\end{array}\right] \times \ldots \\
& \times \exp \left\{j 2 \pi(\tilde{p}-p) d_{\mathrm{Tx}} \sin \theta_{\mathrm{Tx}} \cos \phi_{\mathrm{Tx}} / \lambda\right\} \\
& \times \exp \left\{-j 2 \pi f_{\mathrm{Tx}} \xi \sin \theta_{\mathrm{Tx}} \cos \left(\phi_{\mathrm{Tx}}-\gamma_{\mathrm{Tx}}\right)\right\} \\
& \times p(\gamma \mathrm{Tx}) p\left(\Omega_{\mathrm{Tx}}\right) d \gamma_{\mathrm{Tx}} d \Omega_{\mathrm{Tx}}
\end{aligned}
$$

2) Receive spatial correlation: $R_{q \cdot, \tilde{q} \cdot}(\xi)$

$$
\begin{aligned}
& R_{\cdot q, \tilde{q}}(\xi)=\iint\left[\begin{array}{c}
G_{q, \theta} \cdot G_{\tilde{q}, \theta} \\
G_{q, \phi} \cdot G_{\tilde{q}, \phi}
\end{array}\right] \times \ldots \\
& \times \exp \left\{j 2 \pi(\tilde{q}-q) d_{\mathrm{Rx}} \sin \theta_{\mathrm{Rx}} \cos \phi_{\mathrm{Rx}} / \lambda\right\} \\
& \times \exp \left\{-j 2 \pi f_{\mathrm{Rx}} \xi \sin \theta_{\mathrm{Rx}} \cos \left(\phi_{\mathrm{Rx}}-\gamma_{\mathrm{Rx}}\right)\right\} \\
& \times p\left(\gamma_{\mathrm{Rx}}\right) p\left(\Omega_{\mathrm{Rx}}\right) d \gamma_{\mathrm{Rx}} d \Omega_{\mathrm{Rx}}
\end{aligned}
$$

The probability distribution of the scatterers around the transmitter and the probability distribution of scatterers around the receiver are respectively denoted by $p\left(\Omega_{\mathrm{Tx}}\right)$ and $p\left(\Omega_{\mathrm{Rx}}\right)$. We assume that the probability density of the azimuthal and the elevation components are independent.

- $p\left(\Omega_{\mathrm{Tx}}\right)=p\left(\theta_{\mathrm{Tx}}, \phi_{\mathrm{Tx}}\right)$

- $p\left(\Omega_{\mathrm{Rx}}\right)=p\left(\theta_{\mathrm{Rx}}, \phi_{\mathrm{Rx}}\right)$

We consider that the azimuth angle follows the Von Mises distribution where the distribution function is expressed as:

$$
p\left(\phi_{\mathrm{Tx}}\right)=\frac{\exp \left\{k_{\mathrm{Tx}} \cos \left(\phi_{\mathrm{Tx}}-\phi_{\text {mean }}\right)\right\}}{2 \pi I_{0}\left(k_{\mathrm{Tx}}\right)}
$$

where:

- $\phi_{\mathrm{Tx}} \in[-\pi, \pi]$.

- $\phi_{\text {mean }}$ is the mean azimuth angle.

- $I_{0}(\cdot)$ is the modified Bessel function of zeroth order.

- $k_{\mathrm{Tx}}$ evaluates the azimuth angle dispersion.

- $\theta_{\mathrm{Tx}} \in\left[\bar{\theta}_{\mathrm{Tx}}-\Delta \theta_{\mathrm{Tx}}, \bar{\theta}_{\mathrm{Tx}}+\Delta \theta_{\mathrm{Tx}}\right], \bar{\theta}_{\mathrm{Tx}}$ is the mean elevation angle and $\Delta \theta_{\mathrm{Tx}}$ is the standard deviation of the angle spread [1]. 
The elevation angle is assumed to follow the uniform distribution where:

$$
p\left(\theta_{\mathrm{Tx}}\right)=\frac{1}{2 \Delta \theta_{\mathrm{Tx}}}
$$

3) Polarization correlation $\Upsilon$ :

$$
\Upsilon=\left[\begin{array}{ll}
1 & \chi \\
\chi & 1
\end{array}\right]
$$

\section{Simulation RESUlts AND COMMENTS}

Once the channel model is introduced and spatial correlation is evaluated, we firstly present in this section the simulation results for the spatial correlation of both the single and the dual polarized MIMO systems. Then, we evaluate the gain in capacity that could be achieved by the use of the dual polarized antennas.

\section{A. Simulation for the spatial correlation}

The concept of dual polarized MIMO system is depicted in Fig. 2. Unlike single polarized MIMO systems, dual polarized MIMO system deploys at both the ends of the communication link orthogonal antennas with different polarizations.

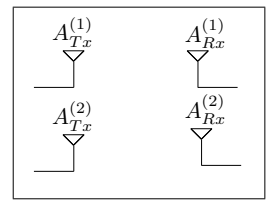

Single polarized

$\operatorname{MIMO}(2 \times 2)$

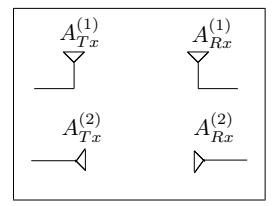

Dual polarized

$\operatorname{MIMO}(2 \times 2)$
Fig. 2. Single polarized versus dual polarized MIMO $(2 \times 2)$

The transmit spatial correlation $R_{\cdot 1, \tilde{2}}(\xi)$ between transmit antennas $A_{T x}^{(1)}$ and $A_{T x}^{(2)}$ is presented in Fig.3. The variation of the spatial correlation for single polarized MIMO system with $\mathrm{z}$ dipole antennas is evaluated in function of the normalized antenna spacing at the transmitter $d_{T x} / \lambda$ and different values of the product $f_{\mathrm{Tx}} \cdot \xi$ in the range of $[0,3]$. For simulations, $k_{\mathrm{Tx}}$ is set to 50 , $\phi_{\text {mean }}$ and $\bar{\theta}_{\mathrm{Tx}}$ equal $90^{\circ}$ and the standard deviation of the angle spread $\Delta \theta_{\mathrm{Tx}}$ is of $90^{\circ}$.

According to the simulation results plotted in Fig.3, the spatial correlation is shown to decay in function of the distance separation between antennas as well as in function of the product $f_{\mathrm{Tx}} \cdot \xi$.

In Fig.4, spatial correlation is presented for dual polarized MIMO system where $\mathrm{z}$ dipole antenna and $\mathrm{x}$ dipole antenna are deployed at the transmitter. The variation of the spatial correlation is presented in function of the mean elevation angle $\phi_{\text {mean }}$ and is shown to decrease as the mean elevation angle approaches $90^{\circ}$ and orthogonality between antennas is conserved.

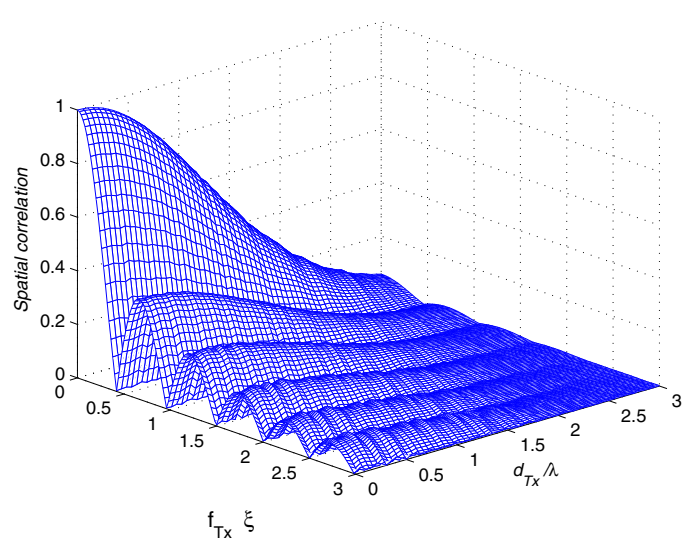

Fig. 3. Spatial correlation for single polarized MIMO system, $R_{\cdot 1, \cdot \tilde{2}}$

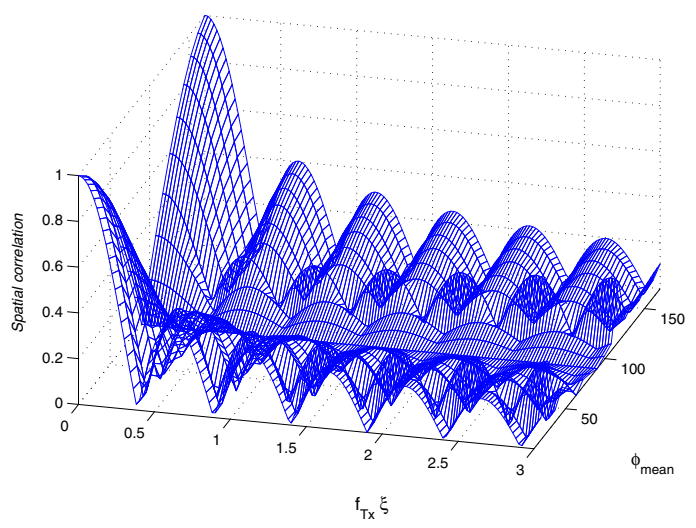

Fig. 4. Spatial correlation at transmit side for dual polarized antennas, $R_{\cdot 1, \cdot \tilde{2}}$

\section{B. Simulation of the capacity gain}

We evaluate the gain in the ergodic capacity obtained as the difference between the dual polarized MIMO system capacity and the capacity of the single polarized MIMO system. The gain in capacity is presented in Fig.5 as a function of the inverse cross polarization discrimination and the spatial correlation between antennas. According to the simulation results, we conclude that the gain in capacity is affected by the mismatch in antenna polarization which is evaluated by the cross polarization discrimination $X P D$. In addition, the gain in capacity with polarized antennas is shown to be more important when antennas at the MIMO system with single polarized antennas have higher correlation. Finally, it is important to notice that the gain in capacity thanks to the use of dual polarized antennas could be only achieved when the cross polarization discrimination could not exceed certain limits. Otherwise, single polarization strategy performs better. 


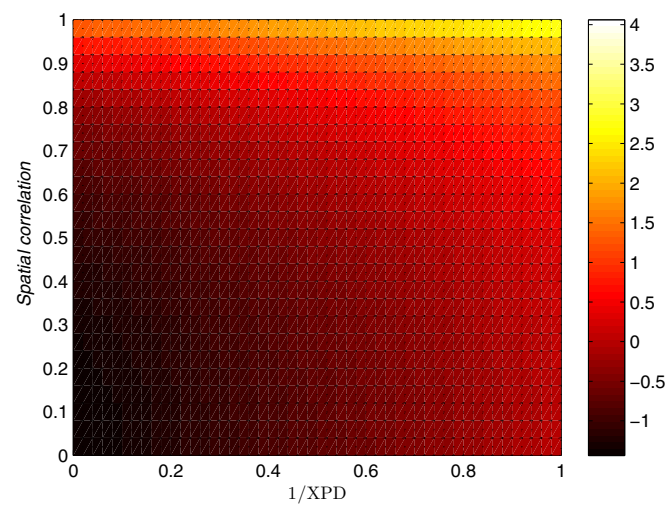

Fig. 5. Gain in capacity of MIMO $(2 \times 2)$ as a function of spatial correlation and the inverse XPD

\section{CONCLUSION}

Throughout this paper, we have presented the wide band channel model for the MIMO system with polarized antennas. We have addressed the phenomena of antenna depolarization in rich scattering environment and evaluated the spatial correlation of antennas. Simulation results for the spatial correlation have been presented for both the single polarized MIMO systems and the dual polarized ones. We have shown in this paper that the gain in the MIMO system capacity achieved with dual polarization technique when comparing to the single polarization strategy depends on the cross polarization discrimination level. In fact, with high levels of the cross polarization discrimination, single polarized MIMO system could perform better. The variation of the gain in capacity as a function of the inverse value of the cross polarization discrimination has been presented for different levels of the spatial correlation. According to the simulation results, we conclude that the gain in capacity becomes more important when higher spatial correlation is measured.

\section{REFERENCES}

[1] K. Raoof, M. Ben Zid, N. Prayongpun, and A. Bouallègue, Advanced MIMO Techniques: Polarization Diversity and Antenna Selection, in: MIMO Systems, Theory and Applications, ISBN 978-953-307-245-6: InTech, April 2011. [Online]. Available: http://www.intechopen.com/articles/show/title/advanced-mimotechniques-polarization-diversity-and-antenna-selection.

[2] K. Raoof and H. Zhou, Advanced MIMO Systems, ISBN : 9781-935068-02-0, USA : Scientific Research Publishing, 2009.

[3] K. Raoof and N. Prayongpun, "Channel capacity performance for MIMO polarized diversity systems," in Proceedings of Wireless Communications, Networking and Mobile Computing (WCNMC), pp. 1-4, December 2005.

[4] K. Raoof and N. Prayongpun, "Impact of depolarization effects on MIMO polarized wireless configuration," in Proceedings of the International Conference on Wireless Communications, Networking and Mobile Computing (WiCOM), pp. 1-4, September 2007.

[5] N. Prayongpun and K. Raoof, "Impact of Depolarization Phenomena on Polarized MIMO Channel Performances," International Journal of Communications, Network and System Sciences (IJCNS), vol.1, no.2, pp.124-129, May 2008.
[6] E. Telatar, "Capacity of Multi-antenna Gaussian Channels," European transactions on telecommunications, vol. 10, 1999, pp. 585-595.

[7] N. Prayongpun, Modélisation et étude de la capacité du canal pour un système multi-antennes avancé exploitant la diversité de polarisation, Thesis dissertation, 2009. 